\title{
Comparison of clinical features and laboratory findings of coronavirus disease 2019 and influenza A and B infections in children: a single-center study
}

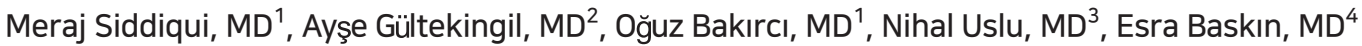 \\ ${ }^{1}$ Department of Pediatrics, Baskent University, Ankara, Turkey; ${ }^{2}$ Department of Pediatric Emergency, Baskent University, Ankara, Turkey; ${ }^{3}$ Department of \\ Radiodiagnostics, Baskent University, Ankara, Turkey; ${ }^{4}$ Department of Pediatric Nephrology and Rheumatology, Baskent University, Ankara, Turkey
}

Background: As the coronavirus disease 2019 (COVID-19) outbreak continues to evolve, it is crucially important for pediatricians to be aware of the differences in demographic and clinical features between COVID-19 and influenza A and B infections.

Purpose: This study analyzed and compared the clinical features and laboratory findings of COVID-19 and influenza A and B infections in children.

Methods: This retrospective study evaluated the medical data of 206 pediatric COVID-19 and 411 pediatric seasonal influenza A or B patients.

Results: COVID-19 patients were older than seasonal influenza patients (median [interquartile range], 7.75 [2-14] years vs. 4 [2-6] years). The frequency of fever and cough in COVID-19 patients was lower than that of seasonal influenza patients ( $80.6 \%$ vs. $94.4 \%, P<0.001$ and $22.8 \%$ vs. $71.5 \%, P<0.001$, respectively). Ageusia (4.9\%) and anosmia (3.4\%) were present in only COVID-19 patients. Leukopenia, lymphopenia, and thrombocytopenia were encountered more frequently in influenza patients than in COVID-19 patients $(22.1 \%$ vs. $8.5 \%$, $P=0.029 ; 17.6 \%$ vs. $5.6 \%, P=0.013$; and $13.2 \%$ vs. $5.6 \%, P=$ 0.048 , respectively). Both groups showed significantly elevated monocyte levels in the complete blood count (70.4\% vs. $69.9 \%$, $P=0.511)$. Major chest $\mathrm{x}$-ray findings in COVID-19 patients included mild diffuse ground-glass opacity and right lower lobe infiltrates. There were no statistically significant intergroup differences in hospitalization or mortality rates; however, the intensive care unit admission rate was higher among COVID-19 patients ( $2.4 \%$ vs. $0.5 \%, P=0.045)$.

Conclusion: In this study, pediatric COVID-19 patients showed a wide range of clinical presentations ranging from asymptomatic/mild to severe illness. We found no intergroup differences in hospitalization rates, oxygen requirements, or hospital length of stay; however, the intensive care unit admission rate was higher among COVID-19 patients.

Keywords: Child, Influenza, Monocyte, SARS-CoV-2 infection

\begin{abstract}
Key message
Question: What are the differences between coronavirus disease 2019 (COVID-19) and influenza infections in children?

Finding: Pediatric COVID-19 patients predominantly exhibited respiratory and/or gastrointestinal symptoms, neurological manifestations, olfactory/gustatory dysfunction, elevated monocytes, mildly elevated C-reactive protein, and unilateral or diffuse abnormalities on chest $\mathrm{x}$-ray. Patients with underlying medical conditions had higher intensive care unit admission rates and should be followed closely.

Meaning: The clinical presentations of pediatric COVID-19 patients varied from asymptomatic/mild to severe.
\end{abstract}

\section{Introduction}

The severe acute respiratory syndrome coronavirus-2 (SARS$\mathrm{CoV}-2$ ) disease is a highly contagious respiratory virus and has affected a large population in less than a year. ${ }^{1)}$ It is suggested that $S$ protein on SARS-Cov-2 binds to the angiotensin-converting enzyme (ACE2) receptor on host epithelial cells and the resultant fusion plays an important role in virus invasion. ${ }^{2)}$ SARS$\mathrm{CoV}-2$ enters the lungs via ACE2 receptor, and mainly invades the alveolar epithelial cells, leading to respiratory symptoms. ${ }^{2)}$ Compared to adults, children often have milder or asymptomatic SARS-CoV-2 infection. ${ }^{3-5)}$ SARS-CoV-2 infection also has some clinical similarities with seasonal respiratory infections in children, especially influenza A and B infections but clinical features are not essentially the same. $\left.{ }^{6}\right)$ Acute respiratory distress syndrome considered a significant disorder of coronavirus disease 2019 (COVID-19) and influenza infections in all age groups. ${ }^{7}$ Severe COVID-19 and influenza infection in previously unexposed young children may lead to alveolar lymphocytes and monocytes infiltration causing acute respiratory distress syndrome. ${ }^{78}$ Early differential diagnosis of COVID-19 and influenza can lead to proper treatment with appropriate medications while reducing the community transmission of COVID-19 infection. Therefore, early differentiation of both infections in the pediatric population

Corresponding author: Meraj Siddiqui, MD. Department of Pediatrics, Baskent University, Ankara, Turkey

凶Email: siddiqui@baskent.edu.tr, https://orcid.org/0000-0002-5739-6590

Received: 19 January, 2021, Revised: 3 May, 2021, Accepted: 7 May, 2021

This is an open-access article distributed under the terms of the Creative Commons Attribution Non-Commercial License (http://creativecommons.org/licenses/by$\mathrm{nc} / 4.0 /$ ) which permits unrestricted non-commercial use, distribution, and reproduction in any medium, provided the original work is properly cited.

Copyright (c) 2021 by The Korean Pediatric Society 
is crucially important during seasons with high infection rates and in low resource settings where diagnostic tests for each viral infection cannot be performed rapidly. Currently, there are few reports comparing the clinical and laboratory features of SARSCoV-2 infection with seasonal influenza viruses in children. The main objective of this study is to analyze and compare the clinical features and laboratory findings of SARS-CoV-2 and seasonal influenza $\mathrm{A}$ and $\mathrm{B}$ infections.

\section{Methods}

\section{Study design and participants}

This retrospective study included the children with either confirmed COVID-19 infection or influenza A and B infection. The first group included children who presented to Baskent University Hospital Pediatric Emergency department between December 2017 and March 2018 with fever and/or respiratory symptoms and tested positive for influenza A or B infection with a fluorescent immunoassay-based rapid influenza antigen test. QuickVue influenza A+B test (Quidel, San Diego, CA, USA) was used to detect the influenza antigens directly from the nasopharyngeal swab specimens. The second group included children who presented to the same emergency unit with fever and/or respiratory complaints with or without contact history between March 12 and December 31, 2020 and tested positive for COVID-19 with real-time reverse transcriptase-polymerase chain reaction test from the nasopharyngeal swab using a certified molecular diagnostic method (Rotor-Gene Q 5plex HRM-Qiagen, Düsseldolf, Germany). The study was approved by the Institutional Review Board of Baskent University (No. KA21/192).

Data regarding the demographic, clinical features and laboratory findings of the patients were obtained from Baskent University Hospital's electronic medical record system. Duration of symptoms, hospitalization, and outcomes was noted, and re-infection and postinfectious complications were reconfirmed from telephone inquiries. According to Dong et al..$^{5)}$ and Fang et al. ${ }^{9)}$ criteria, COVID-19 cases in our study were classified into asymptomatic, mild, moderate, and severe based on clinical presentations, laboratory, and radiologic findings of the patients.

(1) Asymptomatic infection: included patients with confirmed nasopharyngeal COVID-19 RT-PCR test but without any clinical features and laboratory findings.

(2) Mild illness: included patients with fever and/or acute respiratory tract symptoms. Physical examination revealed oropharyngeal congestion but without severe respiratory and imaging findings.

(3) Moderate illness: included patients with symptoms of lower respiratory tract infection. The physical examination can reveal abnormal lung sounds but without tachypnea or hypoxemia. Chest x-ray of the patients showed mild to moderate pneumonic infiltrations.

(4) Severe illness: included patients with symptoms of severe respiratory distress, hypoxemia (oxygen saturation $<92 \%$ ), and radiologic findings (ground-glass opacity and/or consolidation).

(5) Critical illness: included patients with respiratory failure, multiorgan failure, or shock.

Leukopenia and leucocytosis were defined as white blood cells (WBCs) $<4.5 \times 10^{3} / \mu \mathrm{L}$ and $>11 \times 10^{3} / \mu \mathrm{L}$, respectively. Lymphopenia and lymphocytosis were defined as blood lymphocytes $<1 \times 10^{3} / \mu \mathrm{L}$ and $>4 \times 10^{3} / \mu \mathrm{L}$, respectively. Thrombocytopenia and thrombocytosis were defined as blood thrombocytes $<150 \times 10^{3} / \mu \mathrm{L}$ and $>400 \times 10^{3} / \mu \mathrm{L}$, respectively. Monocytosis was defined as blood monocytes $>1 \times 10^{3} / \mu \mathrm{L}$ or $>10 \%$. Mildly elevated C-reactive protein (CRP) was defined as CRP greater than $5 \mathrm{mg} / \mathrm{L}$ but less than $10 \mathrm{mg} / \mathrm{L}$.

CELL-Dyn Ruby or CELL-Dyn 3700 (Abbott Laboratories, Abott Part, IL, USA) hematology analyzer was used for the measurements of WBC, platelets, neutrophil, lymphocyte, and monocyte. For the chest x-ray imaging, SIEMENS Multix Fusion Max system with cesium iodide scintillator was used. Chest x-ray images were interpreted by an experienced radiologist unaware of the diagnosis of the patients. Collected data were reviewed by all authors to verify data accuracy.

\section{Statistical analysis}

Descriptive statistics were presented with frequencies and percentages for discrete variables and with median and interquartile range for continuous variables. Clinical features of COVID-19 group and İnfluenza group were compared by using the Pearson chi-square test and Fisher exact test for categorical variables. Continuous variables were compared using the Student $t$ test. Data were analyzed using IBM SPSS Statistics ver. 22.0 (IBM Co., Armonk, NY, USA). A 2-sided $P<0.05$ was considered statistically significant.

\section{Results}

\section{Baseline clinical features and laboratory findings of COVID- 19 infection}

According to Dong et al. ${ }^{5)}$ and Fang et al. ${ }^{9)}$ criteria, 5 patients (2.4\%) were classified as asymptomatic, 139 (67.5\%) had mild illness, 56 (27.2\%) had a moderate illness, 6 (2.9\%) had severe illness. A total of 206 patients with a median age of 7.55 years (interquartile range, 2-14 years) were tested positive for SARS$\mathrm{CoV}-2$. The main presenting symptoms were as follows; Fever in 166 patients $(80.6 \%)$, cough in $47(22.8 \%)$, sore throat in 45 (21.8\%), runny nose in 40 (19.4\%), headache in $26(12.6 \%)$, fatigue in $22(10.7 \%)$, vomiting in $19(9.2 \%)$, and diarrhea in 9 (4.4\%). Nineteen (9.2\%) patients had underlying chronic conditions (Mainly asthma in 6 [2.9\%], congenital heart disease in 3 [1.4\%], chronic kidney disease in 5 [2.4\%], chronic liver disease in 2 [1\%], and others in 3 [1.4\%]). Epidemiological and clinical characteristics of COVID-19 patients are presented in Table 1. Out of 206 COVID-19 patients, 71 (34.4\%) had 
Table 1. Epidemiologic and clinical characteristics of pediatric COVID-19 versus influenza $A$ and $B$ patients

\begin{tabular}{|c|c|c|c|}
\hline Variable & $\begin{array}{c}\text { SARS-CoV-2 } \\
(n=206)\end{array}$ & $\begin{array}{c}\text { Influenza A \& B } \\
(\mathrm{n}=411)\end{array}$ & $P$ value \\
\hline \multicolumn{4}{|l|}{ Sex } \\
\hline Male & $110(53.4)$ & $227(55.2)$ & 0.365 \\
\hline Female & 96 (46.6) & $184(44.8)$ & \\
\hline Age (yr) & $7.75(2-14)$ & $4(2-6)$ & \\
\hline $0-1$ & $24(11.7)$ & $28(6.8)$ & 0.032 \\
\hline $1-5$ & $58(28.2)$ & $250(60.8)$ & 0.001 \\
\hline $5-10$ & $43(20.9)$ & $102(24.8)$ & 0.161 \\
\hline$>10$ & $81(39.3)$ & $31(7.5)$ & 0.001 \\
\hline \multicolumn{4}{|l|}{ Symptom } \\
\hline Fever & $166(80.6)$ & $388(94.4)$ & 0.001 \\
\hline Cough & $47(22.8)$ & $294(71.5)$ & 0.001 \\
\hline Sore throat & $45(21.8)$ & $68(16.5)$ & 0.108 \\
\hline Runny nose & $40(19.4)$ & $178(43.3)$ & 0.001 \\
\hline Headache & $26(12.6)$ & $16(3.9)$ & 0.001 \\
\hline Vomiting & $19(9.2)$ & $85(20.7)$ & 0.001 \\
\hline Nausea & $7(3.4)$ & $0(0)$ & \\
\hline Abdominal pain & $6(2.9)$ & $28(6.8)$ & 0.045 \\
\hline Diarrhea & $9(4.4)$ & $18(4.4)$ & 0.789 \\
\hline Myalgia & $15(7.3)$ & $12(2.9)$ & 0.012 \\
\hline Arthralgia & $10(4.9)$ & $0(0)$ & \\
\hline Fatigue & $22(10.6)$ & $66(16.1)$ & 0.072 \\
\hline Ageusia & $10(4.9)$ & $0(0)$ & \\
\hline Anosmia & $7(3.4)$ & $0(0)$ & \\
\hline Congested nose & $4(1.9)$ & $0(0)$ & \\
\hline PND & $6(2.9)$ & $0(0)$ & \\
\hline $\mathrm{RD}$ & $4(1.9)$ & $0(0)$ & \\
\hline Poor oral intake & $3(1.5)$ & $0(0)$ & \\
\hline Skin rash & $1(0.5)$ & $0(0)$ & \\
\hline Syncope & $1(0.5)$ & $0(0)$ & \\
\hline Chronic diseases & $19(9.2)$ & $28(6.8)$ & 0.287 \\
\hline Asthma/AR & $6(2.9)$ & $11(2.7)$ & 0.525 \\
\hline $\mathrm{CHD}$ & $3(1.4)$ & $5(1.2)$ & 0.534 \\
\hline CKD & $5(2.4)$ & $4(0.97)$ & 0.144 \\
\hline CLD & $2(1.0)$ & $6(1.4)$ & 0.466 \\
\hline Other(s) & $3(1.4)$ & $2(0.5)$ & 0.210 \\
\hline
\end{tabular}

Values are presented as number (\%) or median (interquartile range). COVID-19, coronavirus disease 2019; SARS-CoV-2, severe acute respiratory syndrome coronavirus-2; PND, postnasal discharge; RD, respiratory distress; AR, allergic rhinitis; CHD, Congenital heart disease; CKD, chronic kidney disease; CLD, chronic liver disease.

Boldface indicates a statistically significant difference with $P<0.05$.

complete blood count and biochemistry tests. Leukopenia and lymphopenia were observed in $6(8.4 \%)$ and 4 patients (5.6\%), respectively. Only 10 out of 71 patients had leucocytosis. Of these patients, monocytosis was detected in 50 patients (70.4\%) which was the only significantly elevated complete blood count parameter in our study. Thrombocytosis and mildly elevated CRP level were observed in $1(1.4 \%)$ and 28 patients (40\%), respectively. Detailed laboratory and imaging findings are shown in Table 2. Seven patients (3.4\%) were hospitalized, and 5 patients $(2.4 \%)$ had thorax computed tomography scanning due to moderate to severe respiratory symptoms. In computed tomography scanning, ground-glass opacity and minimal pleural effusion were common findings. Five patients 5 (2.4\%) were admitted to the intensive care unit (ICU); all had coexisting conditions (such as chronic kidney disease, chronic liver disease, and atrioventricular septal defect). A 7-month-old girl with Down syndrome and congenital atrioventricular septal defect had a multiorgan failure and died 2 weeks after hospitalization.

\section{Baseline clinical features influenza $A$ and $B$ patients}

Of these patients, 312 patients (75.9\%) were diagnosed with influenza A, and 103 (25.1\%) were diagnosed with influenza B. The most frequent presenting symptoms at emergency department were as follows, fever in 388 patients (94.4\%), cough in 294 (71.5\%), sore throat in $68(16.5 \%)$, runny nose in 178 (43.3 $\%)$, headache in 16 (3.9\%), fatigue in 66 (16.1\%), vomiting in 85 (20.7\%), and diarrhea in 18 (4.4\%). Twenty-eight patients (6.8\%) had underlying chronic conditions (major findings were asthma in 11 [2.7\%], congenital heart disease in 5 [1.22\%], chronic kidney disease in 4 [0.97\%], chronic liver disease in 6 [1.4\%], and others in 2 [0.5\%]). Out of 411 influenza patients, $136(33.0 \%)$ had complete blood count and biochemistry tests. Detailed laboratory findings and outcomes of hospitalized patients are shown in Tables 2 and 3 , respectively.

\section{Comparison of clinical features and laboratory findings between COVID-19 and influenza $A$ and $B$ infections}

In our study, no significant differences were found between COVID-19 and influenza cases about gender and comorbidities. For both infectious disease asthma, allergic rhinitis, congenital heart disease, chronic kidney disease, and chronic liver disease were the most common comorbidities. The frequency of patients diagnosed with COVID-19 was older than seasonal influenza patients. In our study, $39.3 \%$ of COVID-19 patients were age $>10$ years which is 5 times higher than the influenza cases $(39.3 \%$ vs. $7.5 \%, P<0.001)$. For both infectious disease fever and/or respiratory complaints were the most common presenting symptoms. Moreover, the frequency of fever, cough and runny nose symptoms in COVID-19 patients were lower than seasonal influenza patients ( $80.6 \%$ vs. $94.4 \%, P<0.001$; $22.8 \%$ vs. $71.5 \%, P<0.001$; and $19.4 \%$ vs $43.3 \%, P<0.001$; respectively). The frequency of patients with a headache and myalgia were more in COVID-19 than seasonal influenza (12.6 $\%$ vs $3.9 \%, P<0.001$ and $7.3 \%$ vs $2.9 \%, P=0.012$ : respectively). Ageusia 10 (4.9\%), anosmia 7 (3.4\%), and arthralgia 10 (4.9 $\%)$ were only reported in COVID-19 patients. Leukopenia and lymphopenia were more frequently observed in seasonal influenza patients rather than COVID-19 patients $(22.1 \%$ vs. $8.5 \%$, $P=0.029 ; 17.6 \%$ vs. $5.6 \%, P=0.013)$. Both groups showed mild to moderate CRP elevation. Detailed radiologic findings are shown in Table 2. No significant differences were found in hospitalization rate, oxygenation requirements, and mortality between the 2 groups. However, ICU admission rate was higher in COVID-19 patients, compared with seasonal influenza 
Table 2. Laboratory and imaging findings of pediatric SARSCOV-2 versus influenza $A$ and $B$ patients

\begin{tabular}{|c|c|c|c|}
\hline Variable & $\begin{array}{c}\text { SARS-CoV-2 } \\
(n=71)\end{array}$ & $\begin{array}{c}\text { Infleunza A \& B } \\
(n=136)\end{array}$ & $P$ value \\
\hline WBC $\left(\times 10^{3} / \mu \mathrm{L}\right)$ & & & 0.029 \\
\hline$<4.5$ & $6(8.5)$ & $30(22.1)$ & \\
\hline $4.5-11^{\text {a) }}$ & $55(77.5)$ & $83(61)$ & \\
\hline$>11$ & $10(14.1)$ & $23(16.9)$ & \\
\hline Platelets $\left(\times 10^{3} / \mu \mathrm{L}\right)$ & & & 0.048 \\
\hline$<150$ & $4(5.6)$ & $18(13.2)$ & \\
\hline $150-400^{a)}$ & $66(93.0)$ & $109(80.1)$ & \\
\hline$>400$ & $1(1.4)$ & $9(6.6)$ & \\
\hline Neutrophils $\left(\times 10^{3} / \mu \mathrm{L}\right)$ & & & 0.144 \\
\hline$<2$ & $11(15.5)$ & $31(22.8)$ & \\
\hline $2-7.8^{\mathrm{a})}$ & $53(74.6)$ & $83(61.0)$ & \\
\hline$>7.8$ & $7(9.9)$ & $22(16.2)$ & \\
\hline Lymphocytes $\left(\times 10^{3} / \mu \mathrm{L}\right)$ & & & 0.013 \\
\hline$<1$ & $4(5.6)$ & $24(17.6)$ & \\
\hline $1-4^{a)}$ & $53(74.6)$ & $99(72.8)$ & \\
\hline$>4$ & $14(19.7)$ & $13(9.6)$ & \\
\hline Monocytes $\left(\times 10^{3} / \mu \mathrm{L}\right)$ & & & 0.223 \\
\hline $0-1^{\text {a) }}$ & $39(54.9)$ & $83(61.5)$ & \\
\hline$>1$ & $32(45.1)$ & $52(38.5)$ & \\
\hline Neutrophils (\%) & & & 0.074 \\
\hline$<40$ & $16(22.5)$ & $41(30.1)$ & \\
\hline $40-72^{\text {a) }}$ & $47(66.2)$ & $68(50.0)$ & \\
\hline$>72$ & $8(11.3)$ & $27(19.9)$ & \\
\hline Lymphocytes (\%) & & & 0.327 \\
\hline$<20$ & $19(26.8)$ & $46(33.8)$ & \\
\hline $20-46^{a)}$ & $38(53.5)$ & $58(42.6)$ & \\
\hline$>46$ & $14(19.7)$ & $32(23.5)$ & \\
\hline Monocytes (\%) & & & 0.511 \\
\hline$<3$ & $0(0)$ & $0(0)$ & \\
\hline $3-10^{\mathrm{a})}$ & $22(31.0)$ & $41(30.1)$ & \\
\hline$>10$ & $50(70.4)$ & $95(69.9)$ & \\
\hline CRP mg/L & & & 0.550 \\
\hline$\leq 5^{\mathrm{a})}$ & $44(62.0)$ & $84(61.8)$ & \\
\hline$>5$ & $28(40)$ & $52(38.2)$ & \\
\hline Chest $x$-rays & $111(54.5)$ & $146(35.5)$ & \\
\hline Normal & $74(66.7)$ & $96(66.2)$ & 0.523 \\
\hline Mild diffuse ground-glass opacity & $21(18.9)$ & $3(2.1)$ & 0.001 \\
\hline Right lower lobe infiltrates & $14(12.6)$ & $3(2.1)$ & 0.003 \\
\hline Left lower lobe infiltrates & $4(3.6)$ & $4(2.8)$ & 0.914 \\
\hline Bronchovascular markings & $0(0)$ & $2(1.4)$ & 0.461 \\
\hline Right/left hilar infiltrates & $3(2.7)$ & $17(11.7)$ & 0.015 \\
\hline Patchy bilateral infiltrates & $0(0)$ & $9(6.2)$ & 0.023 \\
\hline СТ & $5(2.4)$ & $0(0)$ & \\
\hline GGO/consolidation & $3(1.4)$ & $0(0)$ & \\
\hline Pleural effusion & $2(0.9)$ & $0(0)$ & \\
\hline
\end{tabular}

Values are presented as number (\%).

SARS-CoV-2, severe acute respiratory syndrome coronavirus-2; WBC, white blood cell; CRP, C-reactive protein; CT, computed tomography; GGO, ground-glass opacity.

a) Represents the normal or reference ranges.

Boldface indicates a statistically significant difference with $P<0.05$.
Table 3. Outcomes of hospitalized pediatric SARS-CoV-2 versus seasonal influenza patients

\begin{tabular}{lccc}
\hline Variable & $\begin{array}{c}\text { SARS-CoV-2 } \\
(\mathrm{n}=206)\end{array}$ & $\begin{array}{c}\text { Influenza A \& B } \\
(\mathrm{n}=411)\end{array}$ & $P$ value \\
\hline Hospitalization & $7(3.4)$ & $17(4.1)$ & 0.419 \\
ICU & $5(2.4)$ & $2(0.5)$ & 0.045 \\
Needed MV support & $1(0.5)$ & $0(0.0)$ & 0.334 \\
Oxygen requirements & & & 0.313 \\
$\quad$ Nasal canula/ simple mask & $4(1.9)$ & $15(3.6)$ & \\
High flow nasal canula & $2(1.0)$ & $3(0.7)$ & \\
Hospital stays (day) & $3.5(3-7)$ & $4(2-6)$ & \\
Deaths & $1(0.5)$ & $0(0.0)$ & 0.334 \\
\hline
\end{tabular}

Values are presented as number (\%) or median (interquartile range).

SARS-CoV-2, severe acute respiratory syndrome coronavirus-2; ICU, intensive care unit; MV, mechanical ventilation.

Boldface indicates a statistically significant difference with $P<0.05$.

( $2.4 \%$ vs. $0.5 \%, P=0.045$ ) (Table 3). Chronic kidney failure and congenital heart defects were the most common underlying chronic conditions in patients hospitalized with COVID-19. One COVID-19 patient required mechanical ventilation support due to severe respiratory failure and died 2 weeks after hospitalization. No influenza-associated deaths were observed in our study.

\section{Discussion}

As the COVID-19 outbreak continues to evolve, it will be crucially important for pediatricians to be aware of the simila. rities and differences between COVID-19 and influenza $\mathrm{A}$ and B infections. In this study, we compared the clinical features, laboratory, and imaging findings between the 2 groups. The frequency of fever, cough, and runny nose in children with COVID-19 was less common compared with influenza patients. Both groups presented with gastrointestinal symptoms such as diarrhea and vomiting in our study. Several studies suggest that the presence of gastrointestinal symptoms such as diarrhea in adult COVID-19 patients is an important subjective finding that distinguishes it from other viral illnesses. ${ }^{10,11)}$ Influenza infection is characterized by fever and respiratory tract symptoms in all age groups, however, gastrointestinal symptoms such as diarrhea, and vomiting are often observed among young children. ${ }^{12)}$

Ageusia and anosmia are well-known presenting symptoms in adult COVID-19 patients. ${ }^{13-15)}$ Meanwhile: there are limited reports on olfactory/gustatory dysfunctions in children with COVID-19 infections. ${ }^{16)}$ In our study, ageusia and anosmia were the main presenting symptom in $4.9 \%$ and 3.4\% of COVID-19 patients, respectively, whereas none of these symptoms were present in influenza cases. We had a COVID-19 patient whose main presenting symptom was maculopapular rash with pruritis. There is growing evidence on cutaneous manifestations of COVID-19 in children and adults, with the most common reported manifestations are urticaria, vesicular exanthema, erythematous exanthema, and maculopapular rash. ${ }^{17)}$ Therefore, 
clinicians should be aware of the cutaneous manifestations of COVID-19 in children.

In our study, the COVID-19 infection rate in the 1-5 age group was lower while influenza cases were predominantly 1 to 5 years of age. We found that children with COVID-19 had either mild or moderate clinical presenting symptoms as reported previously. ${ }^{4,9,18)}$ However, the less susceptible, and milder nature of COVID-19 infections in young children remains unclear. Several reports suggest that the heterologous immunity, induced by active immunization of children from birth to 5 years of age, could be the possible reason for the less susceptible and milder nature of COVID-19 infection. ${ }^{19-21)}$ Furthermore, we found that the ICU admission rate was higher in COVID-19 patients with underlying medical conditions, showing that although the clinical presentation of COVID-19 in pediatric patients may be milder, clinical course can be more severe. ${ }^{4,6,9)}$ Therefore, COVID-19 patients with underlying medical conditions such as chronic kidney failure, Down syndrome with congenital heart anomalies and chronic liver failure should be followed carefully.

Abnormalities in chest $\mathrm{x}$-ray such as mild diffuse ground-glass opacity and right lower lobe infiltrates were more often present in COVID-19, compared with influenza patients. Leukopenia and lymphopenia are considered as the cardinal laboratory findings in adult COVID-19 patients and are directly associated with the severity of the disease when present with elevated acute phase reactants such as CRP. ${ }^{22}$ However, leukopenia, lymphopenia, and elevated CRP were more frequent in influenza $A$ and B patients in our study. Therefore, it is difficult to use these parameters for differential diagnosis in pediatric patients. On the other hand, COVID-19 cases with moderate or severe symptoms had markedly elevated monocytes in complete blood count. Monocytes are an essential contributor of the innate immune as well as the adaptive immune system in the human body. ${ }^{23)}$ They are members of the mononuclear phagocytic system and actively participate in the initial inflammatory response against extracellular pathogens. ${ }^{23,24)}$ Recent studies sug. gest the expansion of nonclassical CD14+CD16+ subtype with reduced classical CD14+CD16+ subtype with increasing age in adults. ${ }^{24,25)}$ The initial surge of specific monocyte subsets in peripheral blood count of pediatric COVID-19 patients may be associated with less severe COVID-19 infection in the pediatric population. However further molecular studies are necessary to demonstrate this.

In our study, 400 children (97.1\%) with laboratory-confirmed Influenza A or B infection received twice-daily oseltamivir for 5 days. Their laboratory tests were obtained prior to the initiation of oseltamivir treatment. Macrolides were the mainstay treatment for COVID-19 infection in our study due to their possible immunomodulatory, anti-inflammatory, and antiviral effects shown in previous studies. ${ }^{26,27)}$ Patients were randomly given either azithromycin $(10 \mathrm{mg} / \mathrm{kg} /$ day for 1 day, then $5 \mathrm{mg} / \mathrm{kg} /$ day for 4 days) or clarithromycin (14 mg/kg/day for 7 days). Clinical improvement was observed in $59.3 \%$ of the patients within 24 to 72 hours of starting the macrolides treatment. However, it is not clear whether the clinical improvement was associated with the treatment or self-limiting nature of COVID-19 infection in children. There is limited evidence to support the effectiveness of macrolides treatment in COVID-19 patients, therefore, large studies are needed to determine their effects on COVID-19 patients. ${ }^{27)}$ There were no reports of macrolide-related complications in our study. No antiviral drug formally approved for the pediatric patients therefore we restrained from prescribing it.") However, several antiviral drugs are on trial and require further study before they could be used for pediatric patients.

In conclusion, children with COVID-19 predominantly exhibited respiratory and/or gastrointestinal symptoms, neurological manifestations, olfactory/gustatory dysfunctions, elevated monocytes, mildly elevated CRP, and unilateral or diffuse abnormalities on chest $\mathrm{x}$-ray. Laboratory testing had a limited role in differential diagnosis, however, chest $\mathrm{x}$-ray findings such as mild diffuse ground-glass opacity and lower lobe infiltrates were specific to COVID-19 infections in our study. These findings may contribute to clinicians in early diagnosis and proper management of both viral infections in children.

There are some limitations of this study. We acknowledge that randomized selection of clinical and laboratory features of flu patients, and hospitalization criteria between the doctors may be biased due to the retrospective nature of the study. We also acknowledge the small sample size, retrospective nature, and lack of flow cytometric analysis of monocyte subsets are the main limitations. Therefore, there is a need for prospective and longitudinal multicenter studies and validation of findings in different age groups.

\section{Conflicts of interest}

No potential conflict of interest relevant to this article was reported.

\section{Acknowledgments}

We sincerely thank two anonymous reviewers and associate editor of 'Clinical and Experimental Pediatrics' for their helpful comments on earlier drafts of the manuscript. We also acknowledge Duygu Özbek Siddiqui for reviewing the paper before submission.

\section{ORCID}

Meraj Siddiqui @ https://orcid.org/0000-0002-5739-6590

Nihal Uslu @ https://orcid.org/0000-0002-6733-8669

\section{References}

1. To KK, Tsang OT, Leung WS, Tam AR, Wu TC, Lung DC, et al. Temporal profiles of viral load in posterior oropharyngeal saliva samples and serum antibody responses during infection by SARS-CoV-2: an observational cohort study. Lancet Infect Dis 2020;20:565-74.

2. Wan Y, Shang J, Graham R, Baric RS, Li F. Receptor recognition by the novel coronavirus from Wuhan: an analysis based on decade-long structural studies of SARS. J Virol 2020;94:e00127-20. 
3. Hoang A, Chorath K, Moreira A, Evans M, Burmeister-Morton F, Burmeister F, et al. COVID-19 in 7780 pediatric patients: a systematic review. EClinicalMedicine 2020;24:100433.

4. Lu X, Zhang L, Du H, Zhang J, Li YY, Qu J, et al. SARS-CoV-2 infection in children. NEngl J Med 2020;382:1663-5.

5. Dong Y, Mo Xi, Hu Y, Qi X, Jiang F, Jiang Z, et al. Epidemiology of COVID-19 among children in China. Pediatrics 2020;145:e20200702.

6. Song X, Delaney M, Shah RK, Campos JM, Wessel DL, DeBiasi RL. Comparison of clinical features of COVID-19 vs seasonal influenza A and B in US children. JAMA Netw Open 2020;3:e2020495.

7. Khorramdelazad H, Kazemi MH, Najafi A, Keykhaee M, Zolfaghari Emameh R, Falak R. Immunopathological similarities between COVID19 and influenza: investigating the consequences of Co-infection. Microb Pathog 2021;152:104554.

8. Julkunen I, Melén K, Nyqvist M, Pirhonen J, Sareneva T, Matikainen S. Inflammatory responses in influenza A virus infection. Vaccine 2000;19: S32-7.

9. Society of Pediatrics, Chinese Medical Association; Editorial Board, Chinese Journal of Pediatrics. Recommendations for the diagnosis, prevention and control of the 2019 novel coronavirus infection in children (first interim edition). Zhonghua Er Ke Za Zhi 2020;58:E004. (Chinese).

10. Zayet S, Kadiane-Oussou NJ, Lepiller Q, Zahra H, Royer PY, Toko L, et al. Clinical features of COVID-19 and influenza: a comparative study on Nord Franche-Comte cluster. Microbes Infect 2020;22:481-8.

11. Pedersen CJ, Quinn JV, Rogan DT, Yang S. Factors associated with influenza in an emergency department setting. J Emerg Med 2019;56:478-83.

12. Chan MCW, Lee N, Chan PKS, Leung TF, Sung JJY. Fecal detection of influenza A virus in patients with concurrent respiratory and gastrointestinal symptoms. J Clin Virol 2009;45:208-11.

13. Vargas-Gandica J, Winter D, Schnippe R, Rodriguez-Morales AG, Mondragon J, Escalera-Antezana JP, et al. Ageusia and anosmia, a common sign of SARS-COV-2? A case series from four countries. J Neurovirol 2020;26:785-9.

14. Tong JY, Wong A, Zhu D, Fastenberg JH, Tham T. The prevalence of olfactory and gustatory dysfunction in COVID-19 patients: a systematic review and meta-analysis. Otolaryngol Head Neck Surg 2020;163:3-11.

15. Moein ST, Hashemian SMR, Mansourafshar B, Khorram-Tousi A, Tabarsi P, Doty RL. Smell dysfunction: a biomarker for SARS-COV-2. Int Forum Allergy Rhinol 2020;10:944-55.

16. Qiu C, Cui C, Hautefort C, Haehner A, Zhao J, Yao Q, et al. Olfactory and gustatory dysfunction as an early identifier of COVID-19 in adults and children: an international multicenter study. Otolaryngol Head Neck Surg 2020;163:714-21.

17. Rahimi H, Tehranchinia Z. A comprehensive review of cutaneous manifestations associated with SARS-COV-2. Biomed Res Intl 2020; 2020:1236520.

18. Li Y, Wang H, Wang F, Du H, Liu X, Chen P, et al. Comparison of hospitalized patients with pneumonia caused by SARS-COV-2 and influenza A in children under 5 years. Int J Infect Dis 2020;98:80-3.

19. Guan WJ, Ni ZY, Hu Y, Liang WH, Ou CQ, H JX, et al. Clinical charac teristics of coronavirus disease 2019 in China. N Engl J Med 2020;382: 1708-20.

20. Sarialioglu F, Apak FBB, Haberal M. Can hepatitis a vaccine provide protection against covid-19? Exp Clin Transpl 2020;2:141-3.

21. Beric-Stojsic B, Kalabalik-Hoganson J, Rizzolo D, Roy S. Childhood immunization and covid-19: an early narrative review. Front Public Health 2020;8:587007.

22. Liu X, Zhang R, He G. Hematological findings in coronavirus disease 2019: indications of progression of disease. Ann Hematol 2020;99:14218.

23. Siamon G, Annette P. The mononuclear phagocytic system. Generation of diversity. Front Immunol 2019;10:1893.

24. Pence BD. Severe SARS-COV-2 and aging: are monocytes the key? GeroScience 2020;42:1051-61.

25. Seidler S, Zimmermann HW, Bartneck M, Trautwein C, Tacke F. Agedependent alterations of monocyte subsets and monocyte-related chemokine pathways in healthy adults. BMC Immunol 2011;11:30.

26. Pani A, Lauriola M, Romandini A, Scaglione F. Macrolides and viral infections: focus on azithromycin in COVID-19 pathology. Intl J Antimicrob Agents 2020;56:106053.

27. Venditto VJ, Haydar D, Abdel-Latif A, Gensel JC, Anstead MI, Pitts MG, et al. Immunomodulatory effects of azithromycin revisited: potential applications to COVID-19. Front Immunol 2021;12:574425.

How to cite this article: Siddiqui M, Gültekingil A, Bakırcı O, Uslu N, Baskın E. Comparison of clinical features and laboratory findings of coronavirus disease 2019 and influenza A and B infections in children: a single-center study. Clin Exp Pediatr 2021;64:364-9. https://doi.org/10.3345/cep.2021.00066 